\title{
Hairy Polyp of the Oropharynx: An Unusual Cause of Choking, Feeding Problems in a Newborn
}

\author{
Giridhar Guntreddi ${ }^{1,2,3 *}$, Jayasree Vasudevan Nair ${ }^{4}$, Zahrah Taufique ${ }^{5}$, Swayam P. Nirujogi ${ }^{6}$ \\ ${ }^{1}$ SANFORD Medical Center, Bemidji, MN, USA \\ ${ }^{2}$ Des Moines University, Des Moines, IA, USA \\ ${ }^{3}$ University of Minnesota Medical School, Duluth, MN, USA \\ ${ }^{4}$ Sutter Health, Jackson, CA, USA \\ ${ }^{5}$ Pediatrics ENT/Craniofacial Surgery Fellow, Minneapolis, MN, USA \\ ${ }^{6}$ Family Medicine, PGY-2, Tower Health, Reading Hospital, West Reading, PA, USA \\ Email: *Guntreddig@yahoo.com, drjayasreev@yahoo.com, zahrah.taufique@childrensmn.org,drnirujogi@yahoo.com
}

How to cite this paper: Guntreddi, G. Nair, J.V., Taufique, Z. and Nirujogi, S.P. (2022) Hairy Polyp of the Oropharynx: An Unusual Cause of Choking, Feeding Problems in a Newborn. International Journal of Otolaryngology and Head \& Neck Surgery, $11,75-81$.

https://doi.org/10.4236/ijohns.2022.112008

Received: December 13, 2021

Accepted: February 26, 2022

Published: March 1, 2022

Copyright $\odot 2022$ by author(s) and Scientific Research Publishing Inc. This work is licensed under the Creative Commons Attribution International License (CC BY 4.0).

http://creativecommons.org/licenses/by/4.0/

\begin{abstract}
Hairy polyp is an unusual, rare benign developmental malformation of neonates and infants. Embryologically, they are benign lesions containing of both ectodermal and mesodermal origin. Based on size, location of the lesion, they can produce symptoms such as feeding difficulties, airway obstructions, apneic episodes. We present a case of hairy polyp at Oro/Nasopharynx causing feeding difficulties immediately after birth. We explained the mode of presentation, imaging studies, treatment, and review of literature of congenital hairy polyp/choristoma.
\end{abstract}

\section{Keywords}

HP-Hairy Polyp, MRI-Magnetic Resonance Imaging, CT-Computed Tomography, ET-Endotracheal Tube, SP-Soft Palate, FS-Fibrous Stalk, Choristoma, Stridor, Choking

\section{Introduction}

Hairy polyps are rare benign tumors of neonates and infants, first described by Kelly Brown in 1918. They appear as a sausage like pedunculated masses with a white/gray keratinized epithelium interspersed with hair-bearing areas [1]. They are mostly located in oropharynx and nasopharynx, but can also arise from hard palate, soft palate, tonsils, tongue, eustachian tube and middle ear [2]. They are the most common congenital nasopharyngeal tumors with incidence of 1:40,000 
with a female predominance of 6:1 ratio. These are benign epithelial inclusion cystic lesions resulting from abnormal separation of ectoderm and neuroectoderm along with the lines of embryonic fusion, between third - fifth gestational weeks [3]. Histologically, these are bi-germinal in origin, characterized by skin (ectoderm) covering a (mesodermal) core of adipocytes, smooth muscle, and cartilage. Hairy polyps are not associated with any congenital syndromes and there is no genetic predisposition. Few anomalies such as soft and hard palate clefts, agenesis of uvula and external auricle, ankyloglossia, facial hemihypertrophy, left carotid artery atresia, osteopetrosis are associated with hairy polyps [4]. These lesions mostly present in neonatal period but may occasionally be discovered in childhood. The clinical presentation will depend on the size. Location such as larger lesions can cause upper airway obstruction (50\%), respiratory distress, stridor, feeding difficulties (26\%). Other symptoms are hearing loss, otorrhea, vomiting, earache, bleeding, sleep apnea. Hairy polyp is typically suspected based on clinical examination, imaging by CT/MRI. The treatment involves surgical excision, and the diagnosis is confirmed by histopathological examination of the mass.

\section{Case Report}

A Caucasian baby boy was born by Cesarean section to a G1 P0 mother with normal antenatal history and imaging at 37 weeks 5 days of gestational age. Baby APGAR scores were 8 and 9 at one and five minutes respectively. Baby transitioned well without any issues. Baby was exclusively breast fed since birth and developed recurrent episodes of gagging, choking with feeds within 2 hours of delivery. These episodes were associated with desaturations ranging from $78 \%$ $90 \%$ during choking, gagging episodes. Special care nursery nurse noted repeated episodes of gagging with feeds, and she alerted the physician. On thorough oral examination with a tongue depressor, we found a polypoidal whitish lesion hanging from nasopharynx and occupying the oropharynx. It is approximately $3 \mathrm{~cm}$ in size without any ulcers, hemorrhage. The lesion was surrounded with pooled secretions. Subsequently, the baby was evaluated by the pediatric otorhinolaryngology team. On Flexible laryngoscopy, a large white mass was found that appeared to be pedicled off the left soft palate and left pharyngeal wall. This mass extended inferiorly to the epiglottis. The airway was otherwise open and normal. The true vocal cords were moving symmetrically.

MRI imaging showed a pedunculated mildly heterogenous predominantly high T1, low T2 signal lesion extending from the region of the opening of the left eustachian tube, occupying the left side of nasopharynx and oropharynx. The lesion has indistinct proximal margins, measuring approximately $1.3 \times 2.2 \mathrm{~cm}$ coronal (Figure 1) and $1.1 \mathrm{~cm}$ Antero posteriorly (Figure 2). Signal characteristics indicated that much of the lesion was fatty. Subsequently patient underwent surgical excision of the lesion under general anesthesia. The lesion was on the soft palate and well demarcated from the surrounding mucosa and separate from the eustachian tube, adjacent to it (Figure 3). The lesion was excised en bloc with a 


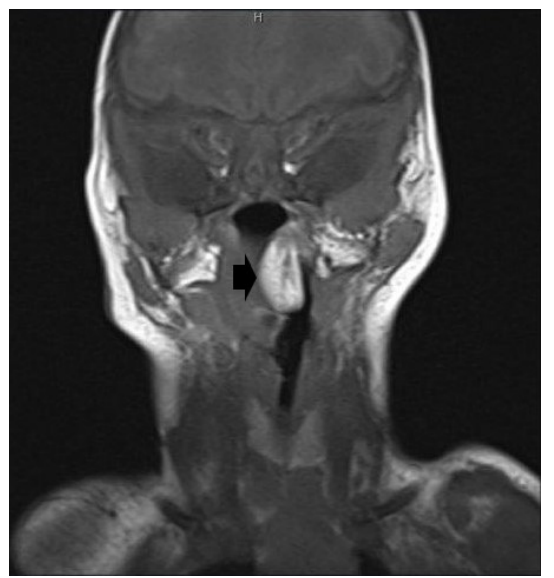

Figure 1. Coronal MRI $1.3 \times 2.2 \mathrm{~cm}$ lesion at post nasal space.

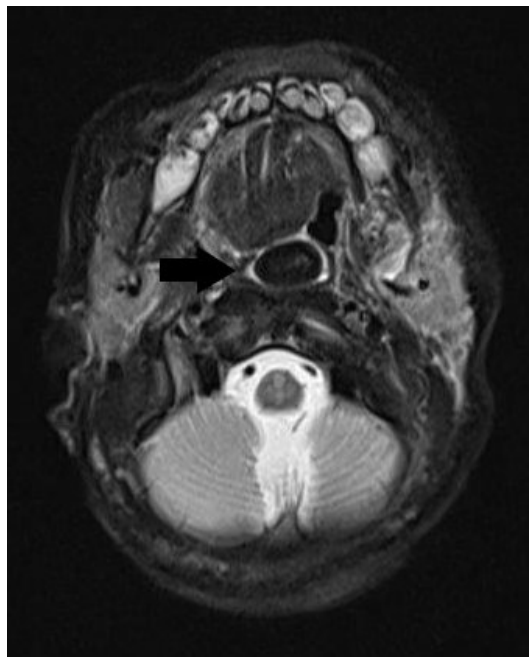

Figure 2. Fat suppressed T2 MRI AP image of the lesion.

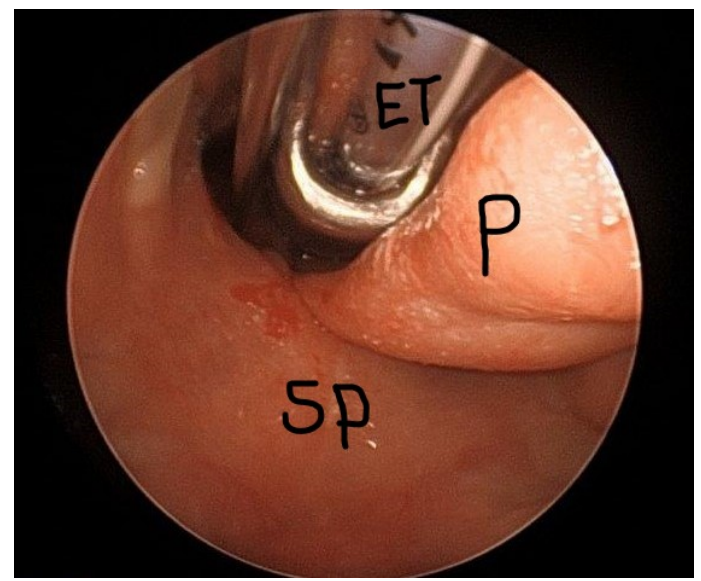

Figure 3. Transoral retraction of the lesion (Polyp - P).

sufficient margin with excellent hemostasis (Figure 4). The post-operative specimen is depicted in the image (Figure 5). Histopathology sectioned showed cross section of polypoid skin tissue with adnexal structures, underlying subcutaneous 


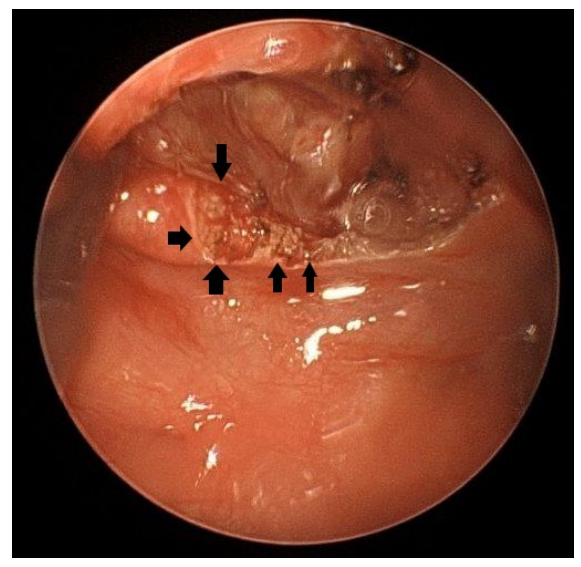

Figure 4. Transnasal view of the defect after the excision.

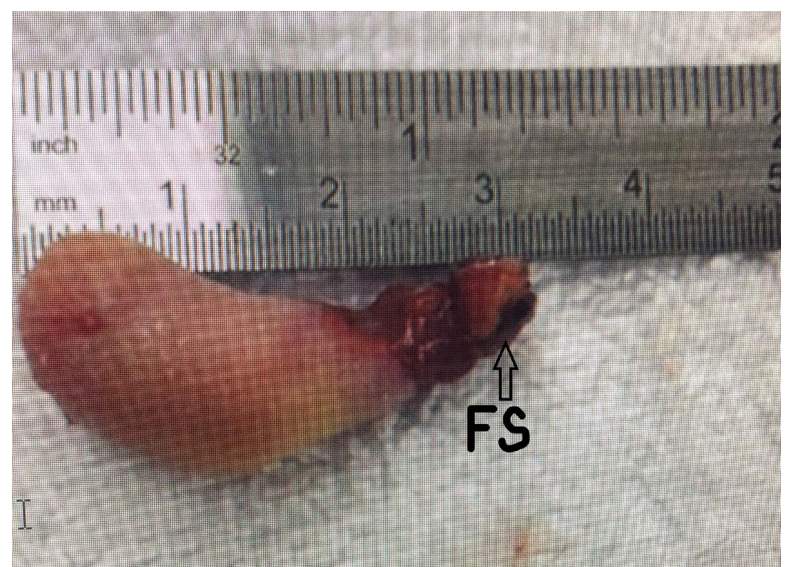

Figure 5. White polypoid fatty mass with fibrous stalk (FS).

fat and centrally located skeletal muscle fibers, fibrous tissue and focal cartilage. Immature/malignant components were not present. The surgical margin revealed non keratinizing squamous to respiratory type epithelium with underlying minor salivary glands, consistent with normal structures at this anatomic location, confirmed as hairy polyp.

\section{Discussion}

Hairy polyp symptoms vary according to the size and location of the lesions. The lesions in nasopharynx, oropharynx usually present as breathing difficulty, snoring, apnea followed by feeding, swallowing difficulties [5]. The typical symptoms are rhinorrhea, snoring, sleep apnea, coughing and gagging. The lesions are sessile/pedunculated ranging from 0.5 to $6 \mathrm{~cm}$ in size and whitish grey/pink in color. When the hairy polyp arises from the eustachian tube, it may cause recurrent purulent otorrhea affecting middle ear ventilation [6]. The apneic episodes may be dangerous and needs urgent intervention, as $25 \%$ of the patients might die before being surgically intervened [7]. Interestingly, a review of presenting hairy polyp diameter and clinical manifestation suggests that hairy polyps of less than $3 \mathrm{~cm}$ in diameter have a higher risk of respiratory distress, as they are more like- 
ly to be missed on routine examination [8].

The diagnosis of hairy polyp is done by characteristic imaging followed by histopathological confirmation. The CT imaging was useful for preoperative delineation of the surrounding bony structures, including the carotid canal, middle ear cavity and ossicles. On CT, they appear as well circumscribed masses containing fat and soft tissue density components. On MRI, hairy polyp demonstrates predominantly hyperintense signal on $\mathrm{T}-1$ and $\mathrm{T}-2$ weighed sequences, with loss of signal fat suppression techniques [9]. The high signal in the T1 W image is the expression of adipose tissue in hairy polyps. Microscopically, these lesions do not pose significant challenge except for closely related entities such as teratoma, hamartoma and dermoid cysts. Teratomas are ruled out by the presence of endodermal derivatives. Hamartomas are ruled out by the presence of single germ layer (usually mesodermal) derivative and dermoid cysts by presence of keratin flakes. Adnexal structures in the skin overlying mesenchymal tissue comprised mostly of fat and cartilaginous nodules as well as central fibrovascular stalk, compatible with hairy polyp. Newer reports also support the hairy polyp is a choristoma or developmental malformation more than teratoma [10]. The differential diagnosis of a neonatal nasopharyngeal mass includes meningoencephalocele, nasal glioma, teratoma, neuroblastoma, hemangioma, foregut thymic, thyroglossal or lingual cyst, Rathke cyst [11].

Treatment of hairy polyp consists of securing the airway first, then completely excising the mass through trans nasal/trans oral/trans tympanic approach [12]. The chances of injury to adjacent eustachian tube, internal carotid artery can be minimized by performing preoperative imaging CT/MRI. Recurrence is very unlikely, and complications are infrequent after surgical excision with excellent hemostasis. Malignant transformation has not been reported so far [13]. Velopharyngeal dysfunction following surgical excision of hairy polyp and postoperative feeding difficulties has been reported in the literature. Our patient never had any intraoperative/post-operative complications. He recovered fully and he is feeding and thriving well.

\section{Conclusion}

The differential diagnosis of obstructive lesions of the upper aerodigestive tract is broad. Hairy polyp can present as an acute cause of respiratory/feeding difficulties. Thorough clinical exam, prompt attentiveness of feeding issues, choking and difficulty with breathing, unstable vitals during feeding alerted the nurse and physician which prompted the further evaluation, through oral exam and subsequent early life saving diagnosis. This case prompts the importance of thorough clinical exam, prompts attention to mother/baby issues for early diagnosis and intervention in rural/sub urban hospital setups. A thorough ear, nose, throat examination should be undertaken in neonates presenting with stridor/respiratory distress/choking/feeding issues. MRI/CT imaging should be undertaken in lesions arising from the postnasal space to exclude intracranial extension and to delineate anatomy to plan for surgery. Complete Surgical excision with excellent 
hemostasis followed by histopathological confirmation of the lesion is the key management of these lesions. The overall prognosis of hairy polyp is excellent [14].

\section{Conflicts of Interest}

The authors declare no conflicts of interest regarding the publication of this paper.

\section{References}

[1] Mc Shane, D., El Sheriff, I., Doyle-Kelly, W., Fennell, G. and Walsh, M. (1989) Dermoids (Hairy Polyps) of the Oro-Nasopharynx. The Journal of Laryngology \& Otology, 103, 612-615. https://doi.org/10.1017/S0022215100109491

[2] Kalcioglu, M.T., Can, S. and Aydin, N.E. (2010) Unusual Case of Soft Palate Hairy Polyp Causing Airway Obstruction and Review of the Literature. Journal of Pediatric Surgery, 45, e5-e8. https://doi.org/10.1016/j.jpedsurg.2010.08.008

[3] Yilmaz, M., Ibrahimov, M., Ozturk, O., Karaman, E. and Aslan, M. (2012) Congenital Hairy Polyp of the Soft Palate. International Journal of Pediatric Otorhinolaryngology, 76, 5-8. https://doi.org/10.1016/j.ijporl.2011.10.008

[4] Burns, B.V., Axon, P.R. and Pahade, A. (2001) Hairy Polyp of the Pharynx in Association with an Ipsilateral Branchial Sinus: Evidence that the Hairy Polyp Is a Second Branchial Arch Malformation. The Journal of Laryngology \& Otology, 115, 145-148. https://doi.org/10.1258/0022215011907569

[5] Dutta, M., Roy, S. and Ghatak, S. (2015) Naso-Oropharyngeal Choriostoma (Hairy Polyp): An Overview and Current Update on Presentation, Management, Origin, and Related Controversies. European Archives of Oto-Rhino-Laryngology, 272, 1047-1059. https://doi.org/10.1007/s00405-014-3050-2

[6] Jin, L. and Zhang, T. (2014) Surgical Treatment of Hairy Polyp in the Eustachian Tube. Chinese Medical Journal, 127, 988-989.

[7] Roh, J.L. (2004) Transoral Endoscopic Resection of a Nasopharyngeal Hairy Polyp. International Journal of Pediatric Otorhinolaryngology, 68, 1087-1090. https://doi.org/10.1016/j.ijporl.2004.03.005

[8] Koike, Y., Uchidia, K., Inoue, M., et al. (2013) Hairy Polyp Can Be Lethal Even When Small in Size. Pediatrics International, 55, 373-376. https://doi.org/10.1111/j.1442-200X.2012.03715.x

[9] Kochanki, S.C., Burton, E.M., Seidel, F.G., Chanin, L.R., Hensley, S. and Acker, J.D. (1990) Neonatal Nasopharyngeal Hairy Polyp: CT and MR Appearance. Journal of Computer Assisted Tomography, 14, 1000-1001. https://doi.org/10.1097/00004728-199011000-00027

[10] Erdogan, S., Tunali, N., Canpolat, T., et al. (2004) Hairy Polyp of the Tongue: A Case Report. Pediatric Surgery International, 20, 881-882. https://doi.org/10.1007/s00383-004-1158-y

[11] Melenie, H. (2008) Tumor Imaging. In: Carachi, R., Grosfeld, J.L., Azmy, A.F., Eds., The Surgery of Childhood Tumors. Springer-Verlag, Berlin, 63-98.

[12] Agrawal, N., Kanabar, D. and Morrison, G.A. (2009) Combined Transoral and Nasendoscopic Resection of an Eustachian Tube Hairy Polyp Causing Neonatal Respiratory Distress. Annals of Otology, Rhinology \& Laryngology, 30, 343-346. https://doi.org/10.1016/j.amjoto.2008.06.001 
[13] Coppit, G., Perkins, J. and Manning, S. (2000) Nasopharyngeal Teratomas and Dermoids: A Review of the Literature and Case Series. International Journal of Pediatric Otorhinolaryngology, 52, 219-227. https://doi.org/10.1016/S0165-5876(00)00288-3

[14] Hulsmann, A.R., de Bont, N., den Hollander, J.C., et al. (2009) Hamartomas of the Oro- and Nasopharyngeal Cavity in Infancy: Two Cases and a Short Review. European Journal of Pediatrics, 168, 999-1001.

https://doi.org/10.1007/s00431-008-0865-0 